\title{
Italique
}

Poésie italienne de la Renaissance

III | 2000

Varia

\section{Fisiologia di un tema boschereccio nel sonetto barocco}

Gian Piero Maragoni

\section{OpenEdition}

Journals

\section{Edizione digitale}

URL: http://journals.openedition.org/italique/191

DOI: 10.4000/italique. 191

ISSN: 1663-4438

\section{Editore}

Librairie Droz

\section{Edizione cartacea}

Data di pubblicazione: 31 dicembre 2000

Paginazione: 89-102

ISBN: 2-600-00490-4

ISSN: 1423-3983

Notizia bibliografica digitale

Gian Piero Maragoni, «Fisiologia di un tema boschereccio nel sonetto barocco », Italique [Online], III | 2000, online dal 06 octobre 2009, consultato il 30 avril 2019. URL : http://journals.openedition.org/ italique/191 ; DOI : 10.4000/italique.191 
Gian Piero Maragoni

F IS I O O G I A I U N TEM A

B O S C H E R E C C I O

N E L S O N E T T O B A R O C C O 
Io mi propongo di fare qualcosa di nuovo in ogni mio pezzo, ma credo di essere sempre stato lo stesso compositore e penso che questo si senta nella mia musica. Ciascun pezzo è un poco differente ma non assolutamente differente.

Győrgy Ligeti 


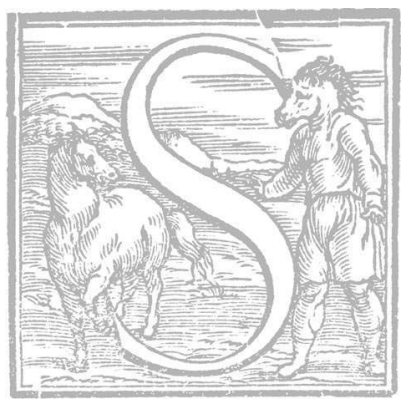

Embrerebbe da ingenui ritenere notevole che in un conte ambientato nell'età del Re Sole (il romanzo Monsieur le jardinier del giovane Richaud al suo debutto) figuri, nascosto in un tal brano odoroso di agraria ed ortaglia:

Sa lutte, il la menait aussi contre [A] les limaces ou les escargots que rebutaient le thym et la sauge, $[B]$ les fourmis qu'éloignaient le sureau ou la capucine, [C] les mouches que dissuadait le basilic, [D] les mouches à miel que contrariaient les fleurs de poires comices, [E] les souris et les campagnols que repoussaient l'euphorbe, le mélilot ou l'oignon. ${ }^{\mathrm{I}}$

un parterre regolato e magnifico, tratteggiato da cinque segmenti compartiti (a decrescere e a crescere) tra la fauna e la flora nemiche:

$$
{ }^{[A] 2 / 2}[B] I / 2{ }_{[C]_{I / I}}^{[D] I+I / I+I}[E] 2 / 3
$$

Inatteso è invece il collier di aggettivi e sostanze aggruppate [(as) (as) (as)S, $A S+A S, A S+A S+A S$ concinnato da Sir Walter Scott sul silvoso limitare dell' Ivanhoe :

Hundreds of broad-headed, short-stemmed, wide-branched oaks, which had witnessed perhaps the stately march of the Roman soldiery, flung their gnarled arms over a thick carpet of the most delicious greensward... ${ }^{2}$

Benché affatto pittoresche e fantastiche, queste veneri di primo Ottocento ci forniscono un esempio tardivo di quel nesso tra retorica e flora ${ }^{3}$ che conserta vegetali e figure o in minuti e lunghissimi elenchi o in sintagmi ordinati $e$ composti per decoro e ornamento, o semiosi. ${ }^{4}$

Il motivo delle piante in sequenza ha per esito figurazioni sigillate (come gli ensembles elaborati a diverso titolo da sottili pareneti e botanici)' o un affrancato ritrarre e descrivere ${ }^{6}$ (costellante e saltuario a piacere). ${ }^{7}$ La flora contemplata in enti singoli può, viceversa, rilevare volta a volta: 
I) o una funzione di narrema, nel racconto

2) o una funzione di figurante, nella similitudine. ${ }^{8}$

Il tema erotico e boschereccio del dono di frutti della terra (quale omaggio od avance dell'amante all'amata ritrosa e restia) si inalveola - per cosi dire esattamente tra $i$ due casi appena adesso definiti, poiché assomma i dinamismi della metafora a falserighe di pretta diegesi.

La ricerca che ora andiamo a tentare non attiene perciò alla tematologia praticata come Ideengeschichte od intesa come storia di un tópos, 9 si ambirebbe a riuscire - piuttosto - la disamina di un tenue motivo nella sua meccanica interna. Di qui - detto da sùbito e in chiaro - la rinuncia a diacronia e diatopia ${ }^{10}$ come limiti e lumi all'inchiesta, e, al contrario, l'aperta preferenza accordata all'indagine in vitro. Se allora la ricerca mancherà in quanto ad attenzione al Sitz im Leben, compensata ad usura sarà essa dal consenso del suo obietto sparuto con la mente ed il cuore barocchi vagheggianti le icone del tiny; l'aver scelto proprio un tema da nulla (fiori in dono come approccio amoroso) poi calato nella forma più piana (il sonetto, e non già il madrigale) sarà infatti già un atto barocco, se è al barocco che meglio si addice sia il culto del minuscolo in estremo ${ }^{11}$ sia il sentimento dell'eventuale reversione tra le massime e le minime delle cose. ${ }^{\mathrm{I} 2}$ Si direbbe in effetti che al genio letterario del nostro Seicento il sonetto sia oggetto di pregio - con un'antimetatesi arguta - in quanto più piccola e squisita tra le forme maggiori e patrizie, e in quanto la più grande e magnanima di quelle minori e fin plebee, onde appunto (come ossimoro in atto : un "picciolo Poema »" ${ }^{\mathrm{I}}$ a doppia faccia) l'essere esso esaltato o depresso con fortune cangianti ed alterne, ${ }^{{ }^{14}}$ sino poi alla nera disistima per il vecchio strumento concepita da tanta cultura poetica degl'indocili secoli entranti. ${ }^{\text {is }}$

La speciale natura del sonetto ${ }^{16}$ potrà farsi palese nel confronto, ed adunque si osservi in che maniera il cavillo del flos interpretatus ${ }^{17}$ (pur presente in Giovanni Palma, Rime, I, 29 : Pianta di garofano inviata a bella Donna, Napoli, I632, p. 38, o in Giovanni Andrea Rovetti, Mormorio d'Elicona, II, 3 : Persico havuto in dono dalla S.D., ${ }^{18}$ Venezia, I630, p. 68, o - con guisa paradossa e antitetica - in Ermes Stampa, Poesie, II, 5: D., che porta fiori in testa, Milano, 1678, p. II9) si biforchi-in Scipione Caetano ${ }^{19}$ - fra due rese non meno che antipodi:

A

Pur hor su 'l crin Corinna mia mi pose

Ghirlanda, a me de la mia stessa vita

Più cara; e quando è di sua mano uscita,

Queste usciron dal cor voci amorose.

«Questa di bianche, e di vermiglie rose

Ghirlanda, ch'ho di propria mano ordita, 
TEMA BOSCHERECCIO NEL SONETTO BAROCCO

Gradisci, Aminta, e mira in lei scolpita La purità de le mie fiamme ascose.

Le vermiglie a te sian del foco ond'ardo Essempio; e l'altre del candor costante De la fede, ch'è in me, ti faccian fede. » Io per la gioia, ch'ogni gioia eccede, Dar sol risposta le potei col guardo, Loquace a un tempo, e taciturno amante.

«Mira, deh mira, o Clori,

Come adorna il bel velo

Del matutino cielo

L'Alba di mille fiori;

Contempla in lei la luce,

Di cui sì ricco è de' tuoi lumi il Sole,

Che tali in te produce

I ligustri, le rose, e le viole.

Mira, mira la terra,

Che 'l ciel mira, e vagheggia ;

Anzi col ciel garreggia,

E fiori apre, e disserra ;

Contempla in lei gli usati

Effetti del tuo piè, da cui dipinti

Son dolcemente i prati

Di narcisi, di gigli, e di giacinti.

Mira quel fior, che nacque

Bianco, e cangiò colore

Per la madre d'Amore,

Per la figlia de l'acque;

Deh contempla in lui quello

Color, che t'orna vagamente il volto,

In cui quanto ha di bello

Per abbellirlo ha la bellezza accolto.

Mira quell'amaranto,

Che di fiore immortale,

Sol perché non è frale,

Ha degnamente il vanto.

Mira, che a lui non cede

Del tuo candido seno il bello eterno,

In cui fiorir si vede

Un April, che non teme ira di Verno. 


\section{Gian Piero Maragoni}

Mira il fiore odorato,

Che del nome d'Aiace

(Mutamente loquace)

È con vaghezza ornato ;

Mira in lui de le ciglia

Tue la sembianza, in cui si vede impresso,

Con altrui meraviglia,

Senza 'l nome d'Amor Amore istesso.

Mira Clitia, che al vago

Lume del Sol, che nasce,

Si rivolge, e si pasce

De l'aspettata imago.

Mira in lei me, che miro

L'albergo tuo, sin ch'io ti veggio; e poi

Sempre intorno m'aggiro

$\mathrm{Al}$ chiarissimo Sol de gli occhi tuoi. »

Così nel vago loco

Che 'l gran Lume d'Etruria in Roma eresse

Discoprendo il suo foco

Le bellezze di Clori Aminta espresse.

In A si ha (I) il serrato racconto di un evento unitario nel tempo (offerta del dono, I-4, e discorso su esso, 5-II ; reazione all'offerta e risposta al discorso, I2-I4); peculiare metrema, per contro, vi appare (2) il beante dischiudersi delle strofe l'una all'altra e fra sé (continuità frasale delle mediane e connessione narrativa delle estreme). Da ciò - nel componimento - un'apoteosi, sia del sonetto quale forma fissa (I) sia del sonetto come vario e duttile tra diverse partizioni a contatto (2).

In $B$, viceversa, la canzonetta quale genere (col suo esser libera nel numero di strofe [I], ma col suo fare isoleggiar ciascuna d'esse [2]) si disposa all'andamento del dettato sia in quanto aperto e addizionale (per rassegna) (I), sia in quanto rigido ed uguale in ogni strofa (2).

Come i polinomî ${ }^{20}$ di B 8 e B I6 (capotasti dei due atrî del carme) sono mise en abyme della lirica quale algebrica enumerazione, così il rispecchiarsi di $B$ 35 in A I4 - stesso ossimoro, ma in ordine inverso - segnala quanto canzone e sonetto sian congiunti nel loro divergere, perché - anche - all'impianto dell'una (voce narrata, I-48, poi voce narrante, 49-52) fa riscontro quello opposto dell'altro (voce narrante, I-4, poi voce narrata, 5-II).

Alla ricerca d'un tal tipo di binarie corrispondenze fra $i$ membri di una raccolta (come studiata successione e compagine) molto si presta il tema del 
dono, riverberante ed inversivo se altro mai. Così, la processione cotematica (quale in Vincenzo Zito, Scherzi lirici, I, 56-57, Napoli, I638, p. 36, o in Giacomo D'Aquino, Rime e prose, 20-23, Napoli, 1638, pp. 81-84) e il diffluire di transiti reciproci (quale in Leonardo Quirini, Vezzi d'Erato, 38 e 54, Venezia, I649, p. 33 e p. 46, o in Pier Francesco Paoli, Rime varie, 53, 72, 79, 103, Roma, 1637, pp. 48, 59, 66, 79):

\begin{tabular}{l|l} 
DONO di lui a lei & DONO di lei a lui \\
\hline DONO di lei a lui & DONO di lui a lei
\end{tabular}

si avvincono nel tópos del regalo, che, se pure in sé solo (da dilemma, ovvero da entimema persuasorio) tende a farsi concetto nel Seicento (come in Antonio Bruni, Le Veneri, I, 9, Roma, I633, p. 33, o in Agostino Augustini, Naturalezze poetiche, I, 68, s.l., s.d., p. 79), ancor più lo diviene allorquando il raddoppio costante del dono volge ad essere effigie fedele dell'amor pastorale perfetto (corrisposto, e pertanto biunivoco ${ }^{21}$ - anziché solitario e infelice).

In codesti due sonetti di Francesco della Valle ${ }^{23}$ due versioni opposte ma omologhe, contrarie però correlative :

\begin{tabular}{l|l} 
A FIORE da lui a lei & B FIORE da lei a lui \\
\hline $\begin{array}{l}\text { A v. \& FIORE subordinato } \\
\text { a lei (più bella) }\end{array}$ & $\begin{array}{l}\text { Av. I4 lei subordinata a FIORE } \\
\text { (più mite) }\end{array}$ \\
\hline B v. 3 convenienza del FIORE & $\begin{array}{l}\text { B v. II convenienza del FIORE a lui } \\
\text { (rorido), non a lei (cocente) }\end{array}$ \\
a lei (bella), non a lui (squallente)
\end{tabular}

si fronteggian salaci ed astute già che l'incipit topico d'epoca ${ }^{24}$ (il deittico ostenso $e$ in iperbato: ${ }^{25}$ vorticante e gestuale, barocco) ${ }^{26}$ le accomuna $e$ distingue al contempo con un destro scambietto di aggiunti:

Questa de le tue labra emula BELLA,

Di colori, e d'odor Rosa GENTILE,

VAGO parto del Ciel, pompa d'Aprile,

Pregio maggior de la stagion novella,

Sole è de' fior, ch'ogn'altro fiore è stella

A paragon di lei basso, et humile ;

Ma riman presso te languida e vile,

Ch'a lei cede ogni fiore, a te cede ella.

Prendila, e fanne fregio al volto, al crine,

Che fra gli ameni suoi vivi colori 
Più splenderan le tue beltà vicine.

Ma come a dar a te gratie, et odori

Colta poco anzi fu da le sue spine,

Lascia le spine tu de' tuoi rigori.

B

Per un fiore havuto dalla S.D.

Questo VAGO, ch'hebb’io, fiore odorato,

De la BELLA tua man dono GENTILE,

Quanto alla rara tua beltà simile,

Tanto diverso è da l'humil mio stato :

Nel tuo bel volto quasi in nobil prato

De la più vaga età fiorisce Aprile ;

De gli anni in me l'Inverno horrido e vile

Mostra che sono a sospirar sol nato.

Il presi pur, ch'a me doveasi alquanto,

Che se fora al tuo sguardo arso, e distrutto,

Io verde il manterrò col rio del pianto.

Fia forse dopo il mio verno di lutto

Primavera di gioia, e spero intanto

Dopo i fior di beltà, d'Amor il frutto.

Alla legge del doppio non sfugge Melosio nel proprio canzoniere ${ }^{27}$, Dona alcune spade di Corallo alla S.D. (I, 8, V); B. Donna dona all'Amante una treccia di Capelli $(I, 8, X I I I)$, soprattutto nel secondo sonetto conseguendo alcunché di notevole :

Signora, il regalarmi alla Spagnuola

Con catenella d'intrecciati crini

È certo favorazzo da Zerbini,

Che son pieni di vento, e 'l vento vola.

Io che non hebbi mai vitio di gola,

Né son di questi amanti soprafini,

Non vado dietro a tanti Moscardini,

E mi contenta una vivanda sola.

Quella treccia è d'un or che non ha spaccio,

Ch'al foco non s'affina, anzi s'affuma.

O che brutto veder portata al braccio!

E non mi state a dir che si costuma,

Perch'io per trarmi d'amoroso impaccio

Ho bisogno di carne, e non di piuma. 
Se in altri (come il Michiele madrigalista de La Benda di Cupido, e.g. I, II4, III, III, Venezia, I648, p. 63 e p. 303, alle prese col diverso motivo del regalo ricusato o spregiato) si tentan variazioni di passo narrativo, qui si applica Melosio ad un degrado di rango e caratura del sonetto, dalla retorica del dolce $e$ dilicato a quella del faceto e del burlesco. Della quale maestrevoli colpi appaiono, nel vivo del carme, certo fischiante ed irto allitterare ("Ch'al foco non S'AFFinA, anzi S'AFFumA»), il comico concerto delle rime (sovracute -ini, biscupe -uma o in intensa-accio), il lessico guascone $e$ picaresco (da alterati e pennacchî disposti in sintagmi rimanti e baciati «catenella d'intrecciati crini : certo favorazzo da Zerbini», "AmANTI soprafini : A tANTI Moscardini », a un regime transitivo integrato "regalarmi... / Con... ", da romanzo dimesso e preciso " ...quattrinelli...che aveva presi quella sera, con intenzione di regalar generosamente don Abbondio... », Promessi Sposi, $I X, 2$ ), la si libera e amena sintassi di una vivida inezia qual l'e (dall'epanortosi con copulativo di rinforzo "di carne, e non di piuma», alla reduplicatio coordinata su cesura «son pieni di vento, e 'l vento vola ", alla congiunzione in esordio totale con un frequentativo fraseologico « $\mathrm{E}$ non mi state a dir »). ${ }^{28}$

Del rimanente, per attizzare un motivo che infine rischî di farsi, o parere, frustraneo (sia che l'omaggio non sortisca l'effetto, sia che, ricambiato, il cerchio si chiuda), è mestieri al poeta di agire:

I) o innovando l'oggetto del dono (surrogato con altro diverso)

2) o ampliandone i virtuali rapporti (per industre contaminazione).

Al primo capo vorranno essere ascritti sonetti quali uno di Baldassarre Pisani, Dona un Pappagallo alla S.N. ${ }^{29}$ ed uno di Filippo Massini, Dono predace ${ }^{30}$ sul regalo - pur campestre - dell'uccello. Al secondo potrei ricondurre questa summa di temi amorosi (bella mano guantata e donante) dovuta a Marcello Macedonio:

Mano donatrice di guanti

O Bella man, ch'i miei desiri affreni, Scarsa al conforto, e prodiga al dolore, Tu nel bel regno del monarca Amore Libero scettro imperiosa tieni.

Tu reggi l'arco al pargoletto, e meni Campion, ch'è cieco, a ritrovarmi il core, E tu avventi i suoi dardi, e tu l'honore De l'antiche vittorie a lui mantieni.

Dolci arnesi di lei, di cui ragiono, 


\section{Gian Piero Maragoni}

Guanti, io vi bagno, e voi m'incenerite;

Voi la spoglia di Nesso, Alcide io sono.

Pur è fredda colei, da cui venite,

Neve è la donatrice, e foco il dono,

E da mano di ghiaccio ardenti uscite. ${ }^{31}$

Qui, come ad essere invocata non è già la ninfa ma una parte d'essa (apostrofe alla mano, I-8), cosi la cosa donata pertiene a quella stessa parte anziché alla donna tutta (apostrofe ai guanti, 9-I4). E per ciò che la topica antitesi predicante la donna spietata (bella, però crudele) ora vien riferita alla mano (Ia / 4b), talché altre su essa si affoltano (2, IO, I3-I4) con piedritti di isocoli (2, I3) e chiasmi (IO, II), e collassi di ossimori in nuce (5, 6).

Inodore corolle d'inchiostro son sfilate finora, mestissime. Non ne incolpi il discreto lettore se non chi, malprudente, ha assemblato presumendo di render servigio con cesoie anziché con sarchielli.

Gian Piero Maragoni 
TEMA BOSCHERECCIO NEL SONETTO BAROCCO

I. Frédéric Richaud, Monsieur le jardinier, XIV, 3, V, Paris, Grasset, I 999, p. I 24.

2. Walter Scott, Ivanhoe, I, 6, Edinburg, Adam \& Charles Black, i87 I, p. 24.

3. Cfr., oltre a V. Cian, Del significato dei colori e dei fiori nel Rinascimento italiano, in Scritti di erudizione e di storia letteraria, Siena, I95 I, p. 32, A. Lanza, La letteratura tardogotica. Arte e poesia a Firenze e a Siena nell'autunno del Medioevo, Anzio, I994, pp. 669-673 e S. Benedetti, Fra dramma e poema. Per una lettura del "Tempio d'Amore» di Galeotto del Carretto, "Rivista di letteratura italiana », I6 (I998), I-3, p. 23 n. 38.

4. L'opposizione salterà all'occhio di chiunque, se confronti l'architetto Bracciolini, Lo scherno degli Dei, III, 56, f :

$$
\text { DI quercia IN mirto, e DI ginepro IN orno; }
$$

con il Monti persuasore oculato nell'età della requie anelata, Per la pace conchiusa tra Francia ed Austria, Napoli, Spagna, 9-I 4 :

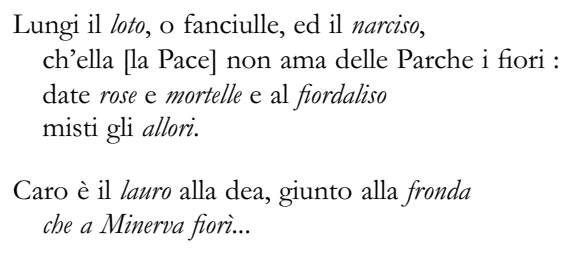

5. Cfr. J.-P. Albert, Odeurs de sainteté. La mythologie chrétienne des aromates, Paris, I 996 (I $990^{\text {I }}$ ), p. 209, G. Leoni, Christ the Gardener and the chain of symbols: the gardens around the walls of sixteenth-century Ferrara, in Aa. Vv., The Italian Garden. Art, design and culture, Cambridge, I996, pp. 74-75 ; M. Zoppi, Storia del giardino europeo, Roma-Bari, I995, p. 8 I.

6. V.D.S. Lichacčev, La poesia dei giardini. Per una semantica degli stili dei giardini e dei parchi, Torino, I 996, p. 333 e F. Pizzoni, Il giardino. Arte e storia, Milano, I997, pp. i 8 5-i 86 ; cfr. et Aa. Vv., Parchi letterari dell'Ottocento, Venezia, I998, p. I 8 vs. A. Fariello, I giardini nella letteratura. Dal giardino classico al giardino romantico, Roma, I 998, p. I 2 I.

7. Similmente al distinguo operabile tra larghissima suggestione campestre (come nella Música para un jardín di Rodrigo o nel Garden concerto di Sauguet) e precisa memoria bucolica, sia pur certo rifratta o corretta (le Georgiche dadaiste di Milhaud, Catalogue de fleurs, i 920, od i numi oxoniensi di Lambert, Pomona, I927).

8. Cfr., oltre a R. de Gourmont, Retorica e stile, Firenze, I995, pp. 4I e 45-46, L. Bornscheuer, Retorica e paradigmi antropologici, Modena, I99 I, pp. 3 I-32 e F. Goyet, Le sublime du "lieu commun ». L'invention rhétorique dans l'Antiquité et à la Renaissance, Paris, I 996, pp. 591-596.

9. Penso ai due bellissimi studî (benché molto diversi tra sé) di F. D’Intino, Il poeta e la tecnica. Le immagini della cera e del reliquiario in Leopardi e Wordsworth, in Aa. Vv., Romanticismo europeo e traduzione. Atti del seminario internazionale, s.l., I995, pp. 269-282 e R. Gigliucci, "Qualis coena tamen!". Il topos anticortigiano del " tinello", "Lettere Italiane ", 50 (I998), 4, pp. 587-605.

ı. Se ne vedano recenti applicazioni non solo in E. Bellini, Umanisti e Lincei. Letteratura e scienza a Roma nell'età di Galileo, Padova, I 997 e D. Chiodo, Suaviter Parthenope canit. Per ripensare la 'geografia e storia' della letteratura italiana, Soveria Mannelli, I999, ma anche in 


\section{Gian Piero Maragoni}

G. Baffetti, Retorica e scienza. Cultura gesuitica e Seicento italiano, Bologna, I997, p. I 50 e M. Sarnelli, "Col discreto pennel d'alta eloquenza ». "Meraviglioso» e Classico nella tragedia (e tragicommedia) italiana del Cinque-Seicento, Roma, 1999, Pp. 170-17 I.

I I. Cfr. G. Pozzi, Presentazione, in Aa. Vv., Petits thèmes littéraires, Fribourg, I986, pp. I 3-I 5 e M. Pieri, Civiltà dei «minori», «L’Informatore Librario », 8 (1978), 7, pp. 17-19.

I 2. Cfr. G. Faure, Storia del microscopio, Roma, I945, pp. I9-2 I e V. Ronchi, Il cannocchiale di Galileo e la scienza del Seicento, Torino, 1958, pp. 76-77.

I 3. Federigo Meninni, Il Ritratto del Sonetto e della Canzone, Venezia, i678, p. iо.

i4. Si riscontrino gli opposti apprezzamenti di un CRistoforo Castelletti (Stravaganze d'Amore, i, 5) e di un Marino (Lettere, I, I 32).

i s. Trascorrendo da un Metastasio criticissimo (ed. Brunelli, IV, p. 506) al Leopardi assai netto detrattore (ed. Binni e Ghidetti, II, p. 300, col. I).

16. Sul trovato spiritoso del quale, come lirica di classe bucolica promanante dal motivo del dono, si vedano le accorte deduzioni di A. Martini, L'invention de la lyrique pastorale entre le Tasse et Marino, «Les Cahiers Forell», 1997, 8, pp. 66-68; cfr. et C. Caruso, Dalla pastorale al poema : l" Adone» di Giovan Battista Marino, in Aa. Vv., La poesia pastorale nel Rinascimento, Padova, I998, p. 363.

17. Cfr. G.P. Maragoni, Tra verzieri ed erbarî. Nota sulle rime boscherecce di Paolo Zazzaroni, «Italica », 73 (1996), 3, pp. 337 e 339 .

I8. Menzione a parte merita il sonetto per qualcosa del suo esastico ultimo :

Dal Sol nudrito de' begli occhi erranti,

Da l'aura de le due labra ridenti,

E da l'onde talhor de' miei gran pianti ;

S'ei [il pesco] non vien manco de' sospiri a i venti,

Crescendo, mostrerà ne' Frutti i vanti

De le bellezze tue, de' miei tormenti.

Esso infatti si smarca ed eccelle (certo più che per l'acuta letteralizzazione - e conseguente iperbole ingegnosa - della grammatica figurale del petrarchismo) per l'unione della sintassi a colata e di due rime semiidentiche in dentale ( $\operatorname{err} a N T i$ : rideNTi : piaNTi : veNTi : vaNTi: tormeNTi») con la messa in valore del morfema che è proprio del modo gerundio ( oNDe-cresceNDo »). Ne sortisce un incesso litanico un po' affine - sebben di lontano all'appiombo dei versi infinitivi aggiustati dal DanTE elegiaste (Purg. 4, I4; 5, 55; 7, I05; 23, 20), presso i quali le ondose sonore (Purg. 5, 24; I3, 3; 23, 17; 25, I26) e l'appeal nominale del verbo (Purg. I 3,$26 ; 25$, I 24) han per esito il vago e il suado (Purg. 5, 39; 7 , I08; I 2, I 20) in contesti obliosi o compunti (Purg. 3, 81 ; I 2, I 36). Sarà bello, a riguardo, il confronto tra gli accenni - già fermi - d'un vate (G. A. Scartazzini, Scritti danteschi, Locarno, 1997, pp. I 24 e 1 38) e gli sviluppi di dantologi recenti (Aa. Vv., Memoria biblica nell'opera di Dante, Roma, 1996, pp. 91-92 ; R. Scrivano, Dante, Commedia. Le forme delloltretomba, Roma, I997, pp. 80-81 ; C. Bologna, Il ritorno di Beatrice. Simmetrie dantesche fra "Vita nova ", "petrose" $e$ «Commedia », Roma, I998, p. 89).

19. Rime I46 e 55, Viterbo, I6 12, pp. 94 е 33-34.

20. Si tratta di quel genere di verso che, offrendosi da accumulo uniforme, finisce per incarnare l'idea di una serie ordinata e infinibile. Di qui (data l'impressione di un occhio che 
TEMA BOSCHERECCIO NEL SONETTO BAROCCO

discorra dal vicino al lontano contemplando infiniti spettacoli sino al filo dell'ampio orizzonte) la clamante funzione anascopica di coacervi, polisindeti e filze :

Col guardo luminoso

Quanto più gradi ASCENDO

Dal confin di Levante,

Più distinti comprendo

Colli, Boschi, Campagne, Huomini, e Piante.

(Lemene, Endimione, III, I, 7-I I)

Fosco di là PROFONDASI

il suol fuggente ai lumi,

e come larve appaiono

città, foreste e fiumi.

(Monti, Al signor di Montgolfier, Iог-104)

SOVRA campagne inargentate ed acque,

Là 've zefiro aleggia,

E mille vaghi aspetti

E ingannevoli obbietti

Fingon l'ombre lontane

Infra l'onde tranquille

E rami e siepi e collinette e ville.

(LeOpardi, Il tramonto della luna, 2-8).

È qua all'opera un'arte avvertita (petrarchistica, insieme, e inventiva : dell'aumento di carica ed aura per dei nomi recati a esser nudi) della quale è fratello germano il moderno sostantivo assoluto, con ufficio descrittorio apparente ed effetto - di fatto - emblematico, o perché lo si privi di articolo contro l'uso e l'attesa di ognuno ("sopra oceano nero », Dino Buzzati, Il deserto dei Tartari, XII, 5 I), o perché il plurale stilistico gli precluda l'antiporta di un Beiwort (« in cumuli ondosi », Guido Morselli, Un dramma borghese, X, I ; «tra lampi del sole », Goffredo Parise, Sillabari, XXII, 33), o perché il predicato predomini, ed inghiotta numerali e conteggî ("c'erano capre infingarde al sole», Elio VitTorini, Conversazione in Sicilia, XXII, 31 ; "V'erano tavoli apparecchiati», VAsco Pratolini, Cronaca familiare, XXVII, 22).

2I. Quello del dono reciproco, che mena alla fusione ed al confondimento, è tema affiorante in Giovanni Canale (Poesie, II, 2, xvi, Venezia, 1667, p. 175), ma è pur lo stesso che al MAnzoni devoto delle Strofe per una prima Comunione $(6,3-4)$ detta - come tesissima resa della mutua oblazione amorosa tra fedele che assume la specie e particola offrentesi a lui una tale sintassi a tarsìa, apparente relitto di arietta :

$$
\begin{aligned}
& \text { Confuso (D) a Te (B) col mio (E) } \\
& \text { Offro (A) il tuo stesso amor (C). }
\end{aligned}
$$

Il settenario tanto incomodo e angusto da costringere a un parlare per capriole è dunque un mezzo contumace e riottoso che il poeta sa mettere a contributo (com'è dei [sons] bouchés presso i maggiori tra i maestri dell'arte di orchestrare, cfr. D. Ceccarossi, Il Corno. Attraverso il suo sviluppo tecnico e coloristico, Milano, 1957, p. 50 sgg. e G. Corti, Il corno, Varese, I998, p. 69), non già spia di un occulto « livello » che zelanti semiologi scovino (ed in tema si veda il dibattito che, civile e proficuo, si aprì tra $\mathrm{M}$. Corti, Il genere «disputatio » e la transcodificazione indolore di Bonvesin da la Riva, "Strumenti critici », 7 (1973), 2-3, pp. 174-178 e G. Orlandi, Letteratura e politica nei «Carmina de mensibus» ("De controversia mensium») di Bonvesin da la Riva, Milano, 1977, pp. 36-37 n. 70). 


\section{Gian Piero Maragoni}

22. Cfr. et V. von Flemming, Arma Amoris. Spracbbild und Bildsprache der Liebe, Mainz, I996, p. 358.

23. Rime, I, го8 е г I 2, Roma, I622, pp. 78 е 80 .

24. Basti il rinvio a Bartolomeo Tortoletti, Rime, III, i 2, i (Roma, i645, p. 274) e Paolo Abriani, Poesie, I, 29, I-2 (Venezia, i664, p. 29).

25. Intorno alla transgressio in Petrarca e nel petrarchismo si potranno cfr. M. Vitale, La lingua del canzoniere ("Rerum Vulgarium Fragmenta») di Francesco Petrarca, Padova, I996, pp. 39I-393, 40 I e R. Bettarini, Lacrime e inchiostro nel canzoniere di Petrarca, Bologna, I998, p. I 3 con I. Pantani, Un'inedita sestina del Trecento (tra le rime di Niccolò Beccari), "Rivista di letteratura italiana ", I I (I993), 3, p. 6ig (nonché N. Gardini, Le umane parole. L'imitazione nella lirica europea del Rinascimento da Bembo a Ben Jonson, Milano, 1997, pp. 82-84 e R. Scrivano, La poesia del Cinquecento, in Storia generale della letteratura italiana, III, Milano, I999, p. 545).

26. Spesso un vezzo di lingua vistoso si propone come un tic, od un'aria - imitabile eppure espressiva - di un'allure dell'ingegno alla moda. Si vedranno, per ciò, i rilievi (sulla voga - in età decadente - dei suffissati in -ale e degli epiteti in in-negativo) di G. Cusatelli, Arturo Graf, in Storia della Letteratura Italiana, VIII, Milano, I968, p. 600 e di R. D’Anna, Diego Angeli narratore, Roma, I998, p. I4.

27. Poesie e prose, Venezia, i695, pp. 3 I 4 e 3 I 8.

28. Per cui si confronti il Parini scommatico di «e non mi state a rompere i... (Alcune poesie di Ripano Eupilino, II, 82, 2).

29. L'armonie feriali, I, 60, Napoli, I695, p. 60.

30. Rime, I, I 53 , Pavia, I609, p. I05. All'ossimoro aguzzo del titolo (per il quale il volatile in dono è altresì un attore di preda) poi sussegue nella lirica il monstrum di uno schema che, rispetto al consueto :

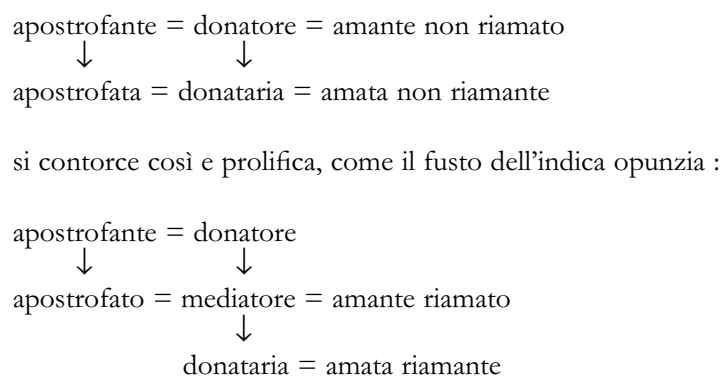

31. Le nove Muse, IV, B, 8, Napoli, I6 14, p. 92. 